\title{
How to tell a gravastar from a black hole
}

\author{
Cecilia B M H Chirenti ${ }^{1,2}$ and Luciano Rezzolla ${ }^{2,3}$ \\ ${ }^{1}$ Instituto de Física, Universidade de São Paulo, São Paulo, Brazil \\ 2 Max-Planck-Institut für Gravitationsphysik, Albert Einstein Institut, 14476 Golm, Germany \\ ${ }^{3}$ Department of Physics, Louisiana State University, Baton Rouge, LA 70803, USA
}

Received 11 June 2007, in final form 29 June 2007

Published 31 July 2007

Online at stacks.iop.org/CQG/24/4191

\begin{abstract}
Gravastars have been recently proposed as potential alternatives to explain the astrophysical phenomenology traditionally associated with black holes, raising the question of whether the two objects can be distinguished at all. Leaving aside the debate about the processes that would lead to the formation of a gravastar and the astronomical evidence in their support, here we address two basic questions: is a gravastar stable against generic perturbations? If it is stable, can an observer distinguish it from a black hole of the same mass? To answer these questions we construct a general class of gravastars and determine the conditions they must satisfy in order to exist as equilibrium solutions of the Einstein equations. For such models we perform a systematic stability analysis against axial perturbations, computing the real and imaginary parts of the eigenfrequencies. Overall, we find that gravastars are stable to axial perturbations, but also that their quasi-normal modes differ from those of a black hole of the same mass and thus can be used to discern, beyond dispute, a gravastar from a black hole.
\end{abstract}

PACS numbers: 04.40.Dg, 04.30.Nk, 04.25.Nx

(Some figures in this article are in colour only in the electronic version)

\section{Introduction}

The gravastar model was proposed by Mazur and Mottola [1] and has attracted attention as a possible alternative to black holes. Within this model, in fact, a massive star in its late stages could end its life as a gravastar, namely a very compact object whose radius would be very close to the Schwarzschild radius (indeed arbitrarily close to it) without having an event horizon or a central singularity. For this to happen, a phase transition is expected to take place at or near the location where the event horizon would have been otherwise formed [2]. The interior of what would have been the black hole is replaced by a suitably chosen portion of de Sitter spacetime with an equation of state (EOS) $p=-\rho$, surrounded by a thin shell of 
ultra-stiff matter with EOS $p=+\rho$, and which is then suitably matched to a Schwarzschild vacuum. In practice, the Mazur-Mottola gravastar model, hereafter the MM model [1], is a spherically symmetric and static five-layer solution of the Einstein equations, including two infinitesimally thin shells needed by the junction conditions of the metric [3].

For as much as it is ingenious, the gravastar model also challenges a building block in modern astronomy, namely the existence of astrophysical black holes. In spite of the many observational evidences in favour of black holes, it may indeed be fundamentally impossible to give an irrefutable observational proof for the existence of a black-hole horizon if only electromagnetic radiation is received [4] and thus tell a black hole apart from a gravastar, if the latter existed. Such a challenge has obviously attracted a lot of attention and several related models have been recently proposed in the attempt to provide answers to two basic questions: once produced, is a gravastar stable to generic perturbations? If stable, can an external observer distinguish a gravastar from a black hole of the same mass?

In [1] it was argued that the MM solution is thermodynamically stable, but other stability analyses are not so easy to perform because of the model's structure. Visser and Wiltshire [5] have analysed the radial stability of a simplified model with three layers and the stability was shown to hold for a number of configurations. This stability was generalized by Carter [6] for gravastar models with different exteriors. Other possibilities for the interior solution have also been considered: Bilić et al [7] replaced the de Sitter interior by a Born-Infeld phantom, while Lobo replaced the interior solution by one that is governed by the dark-energy EOS [8], and Lobo and Arellano matched interior nonlinear electrodynamic geometries to the Schwarzschild exterior [9].

To remove in part the complications produced by the infinitesimal shells in the MM model, Cattoen et al [10] have found that fluid gravastars can be built if the fluid is confined to a given layer and has anisotropic pressures there. The latter essentially replaces the surface tension which was introduced in the original MM model by the matching of the metric in the infinitesimally thin shells. Although anisotropic and with rather arbitrary equations of state, these pressures have the appealing property of being continuous and thus of allowing one to build equilibrium models without the presence of infinitesimally thin shells and thus look more seriously into the issue of stability. Indeed, as a step to going beyond the construction of equilibrium models, DeBenedictis et al [11] made a first attempt to investigate the stability of gravastars through a qualitative analysis of axial perturbations.

In order to provide more definitive answers to the questions mentioned above, we have constructed a general class of fluid gravastars with finite shell thickness and variable compactness. While this class is similar and has been inspired by the one proposed in [10, 11], it also differs in two important aspects. First, while we also consider a fluid gravastar with anisotropic pressures, we try and reproduce the most salient features of the original MM model by creating an internal and an external region which reproduce a de Sitter and a Schwarzschild spacetime at finite radii and not only asymptotically. Second, for these models we determine precise bounds for the properties of the metric functions and the compactness of the gravastar that yield equilibrium solutions, thus considerably restricting the class of possible solutions.

Using these models we have carried out a systematic investigation of the stability analysis of gravastars against axial perturbations [12, 13], thus extending the results discussed in [14] to our gravastar model and computing explicit eigenfrequencies. In this way we were able to conclude that a gravastar is stable to axial perturbations and indeed it is possible to distinguish it from a black hole if gravitational radiation is produced. More specifically, we have found that for all the models considered the imaginary part of the eigenfrequencies is always negative, thus indicating stability against these perturbations. Furthermore, while it is always possible to build a gravastar with given compactness and thickness such that it will have the same 
oscillation frequency as that of a black hole with the same mass, the corresponding decaying time will be different. Our results thus provide a way of distinguishing, observationally and beyond dispute, a gravastar from a black hole.

The paper is organized as follows: in section 2 we review the main features of the original MM model and obtain numerical solutions which enable us to impose bounds for the parameters of the MM solution. In section 3, instead, we present our model for a fluid gravastar with anisotropic pressures and discuss the bounds derived from general conditions imposed by the EOS and the properties of the metric functions. In section 4 , we outline the perturbation equations and the numerical methods employed in the study of axial perturbations of our model. In section 5, we discuss the results obtained for the QNMs and in section 6 we present our final conclusions.

\section{The MM gravastar model}

We first present a quick review on the main features of the original MM model [1] for they will be used in further developments in this paper and highlight that precise bounds exist for the existence of solutions even for the simplest gravastar model.

In general, we consider a static and spherically symmetric line element

$$
\mathrm{d} s^{2}=-f(r) \mathrm{d} t^{2}+\frac{\mathrm{d} r^{2}}{h(r)}+r^{2} \mathrm{~d} \Omega^{2},
$$

and the Einstein equations must be solved for a perfect fluid at rest, such that there are three different regions with the three different equations of state:

$$
\begin{aligned}
& \text { I. Interior: } 0 \leqslant r \leqslant r_{1}, \quad \rho=-p \text {, } \\
& \text { II. Shell: } \quad r_{1} \leqslant r \leqslant r_{2}, \quad \rho=+p \text {, } \\
& \text { III. Exterior: } r_{2} \leqslant r, \quad \rho=p=0 \text {. }
\end{aligned}
$$

In region I, $\rho$ is a constant given by $\rho_{v}=3 H_{0}^{2} / 8 \pi$, and the metric is that of a de Sitter spacetime, so

$$
f(r)=C h(r)=C\left(1-H_{0}^{2} r^{2}\right), \quad 0 \leqslant r \leqslant r_{1},
$$

where $C$ is an integration constant, whose value will be determined later (cf equation (8)).

In region II, a new dimensionless variable $w$ is defined as $w \equiv 8 \pi r^{2} p$, in order to obtain the following set of equations:

$$
\begin{aligned}
\frac{\mathrm{d} r}{r} & =\frac{\mathrm{d} h}{1-w-h}, \\
\frac{\mathrm{d} h}{h} & =-\left(\frac{1-w-h}{1+w-3 h}\right) \frac{\mathrm{d} w}{w}, \\
\frac{w f}{r^{2}} & =\text { const. }
\end{aligned}
$$

An analytical solution can be obtained in the case of a very thin shell, i.e., for $r_{1} \rightarrow r_{2}$. It is easy to see that (4) is satisfied if we take $h=1-\bar{m} / r$ and $\mathrm{d} \bar{m}(r)=2 \mathrm{~d} m(r)=8 \pi \rho r^{2} \mathrm{~d} r^{2}=w \mathrm{~d} r$. Therefore, in the thin-shell limit, (4) can be integrated to yield

$$
h \equiv 1-\frac{\bar{m}}{r} \simeq \epsilon \frac{(1+w)^{2}}{w} \ll 1,
$$

where $\epsilon$ is an integration constant. From the continuity of the metric coefficients $f$ and $h$ at $r_{1}$ and $r_{2}$, it can be shown that the integration constants $\epsilon, C, M$ and $H_{0}$ are given in terms of 
$r_{1}, r_{2}, w_{1}$ and $w_{2}$ by the relations

$$
\begin{aligned}
& \epsilon=-\ln \frac{r_{2}}{r_{1}}\left(\ln \frac{w_{2}}{w_{1}}-\frac{1}{w_{2}}+\frac{1}{w_{1}}\right)^{-1}, \\
& C=\left(\frac{1+w_{2}}{1+w_{1}}\right)^{2}, \\
& M=\frac{r_{2}}{2}\left[1-\frac{\epsilon\left(1+w_{2}\right)^{2}}{w_{2}}\right]=m\left(r_{2}\right), \\
& H_{0}^{2}=\frac{1}{r_{1}^{2}}\left[1-\frac{\epsilon\left(1+w_{1}\right)^{2}}{w_{1}}\right] .
\end{aligned}
$$

Finally, in region III and because of Birkhoff's theorem, the spacetime is described by the Schwarzschild metric,

$$
f(r)=h(r)=1-\frac{2 M}{r}, \quad r_{2} \leqslant r .
$$

Clearly, region II is the most interesting one from a physical point of view since it is where a non-trivial model for the gravastar can be specified. In the case of a 'thick shell', that is away from the thin-shell limit, the gravastar solution in region II can be obtained in the same way as for ordinary spherical stars, namely through the numerical solution of the Tolman-Oppenheimer-Volkoff (TOV) equations for $\rho(r)=p(r)$ and $m(r)$. We recall that in the MM model $\rho(r)$ and $p(r)$ are discontinuous in $r_{1}$ and $r_{2}$ and that $m\left(r_{1}\right)$ must, of course, be less than $r_{1} / 2$.

By specifying $r_{1}, r_{2}$, and thus the shell thickness $\delta \equiv r_{2}-r_{1}$, as well as the initial conditions for the pressure $p\left(r_{1}\right)$ and mass $m\left(r_{1}\right)$, the numerical solution of the TOV equations provides $m(r)$ and $\rho(r)$ for $r_{1}<r \leqslant r_{2}$. A systematic analysis has revealed that a limit exists on the compactness $\mu \equiv M / r_{2}$ of the gravastar and in particular that, for given $r_{1}, r_{2}$ and $m\left(r_{1}\right)$ very close to the limit $r_{1} / 2$, there exists a value for $\rho\left(r_{1}\right)$ which provides the largest $M$. In other words, it is not possible to achieve arbitrary values for $M$. On the other hand, if we fix $r_{2}$ and decrease $r_{1}$ so as to increase the thickness of the shell, we must also decrease $m\left(r_{1}\right)$, and we have verified that the maximum value obtained for $M$ also decreases. Overall, therefore, each shell thickness $\delta$ also selects a maximum compactness of the gravastar $\mu$, above which no solution of the TOV equations can be found.

All of this is shown in figure 1 which reports the space of parameters $(\delta, \mu)$ where equilibrium solutions can be found. The solid curve, in particular, is computed numerically and distinguishes the region where equilibrium models can be found (i.e., the region below the curve), from the region where no solutions can be found (i.e., the region above the curve). Stated differently, for any given shell thickness $\delta$, the solid curve marks the maximum compactness (i.e., the largest value of $\mu$ ) for which gravastar models can be built. Interestingly, therefore, it is not possible to build a very compact gravastar with a very thick matter shell. Rather, gravastars have either large compactness and thin shells, or small compactness and thicker shells, as shown in figure 1. This figure, and its inset in particular, also illustrates that gravastars can be built with arbitrarily large compactness, i.e., with $\mu \rightarrow 1 / 2$ and thus with the outer radius $r_{2}$ being only infinitesimally larger than the corresponding Schwarzschild radius. It is exactly this property that makes gravastars hard to distinguish from a black hole if only electromagnetic radiation is available. 


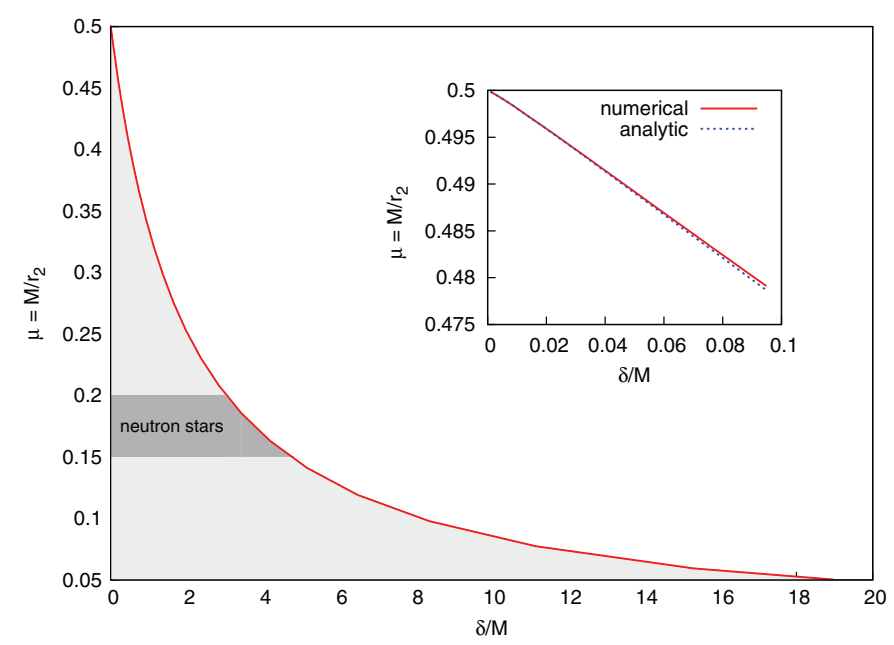

Figure 1. Limits in the compactness and thickness of a gravastar in the MM model. The curve shows the maximum compactness for a given thickness $\delta$ of the shell, that is, in the light grey area below the curve we have the possible solutions, while in the area above the curve solutions are no longer possible. The dark grey area highlights the typical compactnesses for neutron stars. The inset shows a comparison between the numerical solution of the TOV equations with the analytical solution in the thin-shell limit.

\section{The gravastar model with anisotropic pressures}

\subsection{The field equations}

We now discuss our suggestion for a fluid gravastar model in which we follow the spirit of the original MM model and consider a spacetime consisting of three different regions with the internal and external ones reproducing a de Sitter and a Schwarzschild spacetime at finite radii, $r_{1}$ and $r_{2}$, respectively. In addition, we follow the suggestion made in [10] and use a thick shell with a continuous profile of anisotropic pressures to avoid the introduction of infinitesimally thin shells of matter as demanded by the MM [1]. We start therefore with a metric of the form

$$
\mathrm{d} s^{2}=-\mathrm{e}^{v(r)} \mathrm{d} t^{2}+\mathrm{e}^{\lambda(r)} \mathrm{d} r^{2}+r^{2} \mathrm{~d} \Omega^{2},
$$

and a fluid stress-energy tensor $T^{\mu}{ }_{v}=\operatorname{diag}\left[-\rho, p_{r}, p_{t}, p_{t}\right]$, where $p_{r}$ and $p_{t}$ are the radial and tangential pressures, respectively. The Einstein field equations for this spacetime geometry and matter distribution are

$$
\begin{aligned}
& \mathrm{e}^{-\lambda}\left(\frac{\lambda^{\prime}}{r}-\frac{1}{r^{2}}\right)+\frac{1}{r^{2}}=8 \pi \rho, \\
& \mathrm{e}^{-\lambda}\left(\frac{\nu^{\prime}}{r}+\frac{1}{r^{2}}\right)-\frac{1}{r^{2}}=8 \pi p_{r}, \\
& \mathrm{e}^{-\lambda}\left(\frac{\nu^{\prime \prime}}{2}-\frac{\lambda^{\prime} v^{\prime}}{4}+\frac{\nu^{\prime 2}}{4}+\frac{\nu^{\prime}-\lambda^{\prime}}{2 r}\right)=8 \pi p_{t} .
\end{aligned}
$$

It is now convenient to transform the above equations into a form where the hydrodynamical properties of the system are more evident and that reduces to the TOV equations for systems 
with isotropic pressure, i.e.,

$$
\begin{aligned}
& \mathrm{e}^{-\lambda}=1-\frac{2 m(r)}{r}, \\
& v^{\prime}=\frac{2 m(r)+8 \pi r^{3} p_{r}}{r(r-2 m(r))}, \\
& p_{r}^{\prime}=-\left(\rho+p_{r}\right) \frac{v^{\prime}}{2}+\frac{2\left(p_{t}-p_{r}\right)}{r},
\end{aligned}
$$

where

$$
m(r) \equiv \int_{0}^{r} 4 \pi r^{2} \rho \mathrm{d} r .
$$

Combining (18) and (19), we obtain the anisotropic TOV equation

$$
p_{r}^{\prime}=-\left(\rho+p_{r}\right) \frac{m(r)+4 \pi r^{3} p_{r}}{r(r-2 m(r))}+\frac{2\left(p_{t}-p_{r}\right)}{r},
$$

which is reminiscent of the Newtonian hydrostatic-equilibrium equation and where the last term is obviously zero in the case of isotropic pressures, i.e., $p_{t}=p_{r}$.

\subsection{Equation of state}

In order to adopt the model suggested in [10], and still maintain the simple structure of the MM model, we make the following choices for our density function $\rho(r)$ :

$$
\rho(0)=\rho\left(r_{1}\right)=\rho_{0}, \quad \rho\left(r_{2}\right)=0, \quad \rho^{\prime}\left(r_{1}\right)=\rho^{\prime}\left(r_{2}\right)=0 .
$$

A simple way to satisfy the above conditions is to consider a cubic dependence in $r$,

$$
\rho(r)=\left\{\begin{array}{lll}
\rho_{0}, & 0 \leqslant r \leqslant r_{1} & \text { region I } \\
a r^{3}+b r^{2}+c r+d, & r_{1}<r<r_{2} & \text { region II } \\
0, & r_{2} \leqslant r & \text { region III, }
\end{array}\right.
$$

with the coefficients $a, b, c, d$ given by

$$
\begin{aligned}
& a=\frac{2 \rho_{0}}{\left(r_{2}-r_{1}\right)^{3}}, \\
& b=-\frac{3 \rho_{0}\left(r_{2}+r_{1}\right)}{\left(r_{2}-r_{1}\right)^{3}}, \\
& c=\frac{6 \rho_{0} r_{1} r_{2}}{\left(r_{2}-r_{1}\right)^{3}}, \\
& d=\frac{\rho_{0}\left(r_{2}^{3}-3 r_{1} r_{2}^{2}\right)}{\left(r_{2}-r_{1}\right)^{3}} .
\end{aligned}
$$

To obtain the density in terms of the total mass $m\left(r_{2}\right)=M$, we write

$$
\begin{gathered}
\rho_{0}=M \frac{\left(r_{2}-r_{1}\right)^{3}}{4 \pi}\left[\frac{\left(r_{2}^{6}-r_{1}^{6}\right)}{3}-\frac{3\left(r_{2}+r_{1}\right)\left(r_{2}^{5}-r_{1}^{5}\right)}{5}+\frac{3 r_{1} r_{2}\left(r_{2}^{4}-r_{1}^{4}\right)}{2}\right. \\
\left.+\frac{\left(r_{2}^{3}-3 r_{1} r_{2}^{2}\right)\left(r_{2}^{3}-r_{1}^{3}\right)}{3}+\frac{r_{1}^{3}\left(r_{2}-r_{1}\right)^{3}}{3}\right]^{-1} .
\end{gathered}
$$



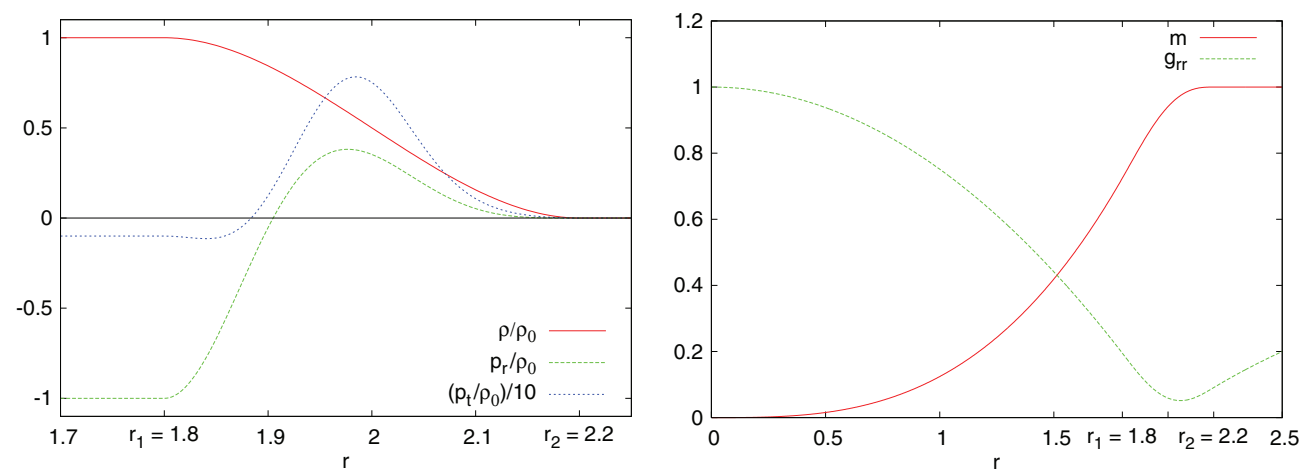

Figure 2. Left panel: behaviour of the functions $\rho(r), p_{r}(r)$ and $p_{t}(r)$ for a representative gravastar model with $M=1, r_{1}=1.8$ and $r_{2}=2.2$. The function $p_{t}(r)$ is scaled by 0.1 for better visualization but provides the dominant contribution in equation (21). Right panel: behaviour of the mass function $m(r)$ and the metric coefficient $g_{r r}$ for the same solution shown in the left panel.

For the radial pressure $p_{r}$, we follow the suggestion made in [11, 15] and use an EOS of the type

$$
p_{r}(\rho)=\left(\frac{\rho^{2}}{\rho_{0}}\right)\left[\alpha-(1+\alpha)\left(\frac{\rho}{\rho_{0}}\right)^{2}\right] .
$$

Clearly, the EOS (29) cannot be derived from basic principles and serves here essentially as a closure relation for the system of the equations. Yet, such an EOS can be constrained and the parameter $\alpha$ is determined by demanding that the maximum sound speed $\mathrm{d}^{2} p_{r} / \mathrm{d} \rho^{2}=0$ coincides with the speed of light to rule out a superluminal behaviour. Because the sound speed

$$
c_{s} \equiv \frac{\mathrm{d} p_{r}}{\mathrm{~d} \rho}=2\left(\frac{\rho}{\rho_{0}}\right)\left[\alpha-2(1+\alpha)\left(\frac{\rho}{\rho_{0}}\right)^{2}\right]
$$

has a maximum for $\bar{\rho}=\rho_{0} \sqrt{\alpha /(2(1+\alpha))}$, requiring $c_{s}(\bar{\rho})=1$ yields $\alpha \simeq 2.2135$. Despite being not particularly realistic, the EOS (29) has the advantage of being simple and possessing the needed physical limits since $p_{r} \rightarrow-\rho$ for $r \rightarrow r_{1}$ and $p_{r} \rightarrow 0$ for $r \rightarrow r_{2}$ (cf the right panel of figure 2 for a typical gravastar model).

Once the solutions for the energy density and the radial pressure are known, the tangential pressure $p_{t}$ can be computed through the anisotropic TOV equation (21) as

$$
p_{t}=p_{r}+\frac{r}{2} p_{r}^{\prime}+\frac{1}{2}\left(p_{r}+\rho\right)\left[\frac{m(r)+4 \pi r^{3} p_{r}}{r(1-2 m(r) / r)}\right] \text {. }
$$

The set of equations (22)-(31) fully determines our gravastar model which will have a finite core described by the de Sitter metric, a crust of matter and an exterior described by the Schwarzschild solution.

The behaviour of the energy density and that of the pressures is shown in the left panel of figure 2 for a representative gravastar model with $M=1, r_{1}=1.8 M$ and $r_{2}=2.2 M$. The right panel in the same figure, instead, shows the mass function and the metric coefficient $g_{r r}$ for the same representative model. 

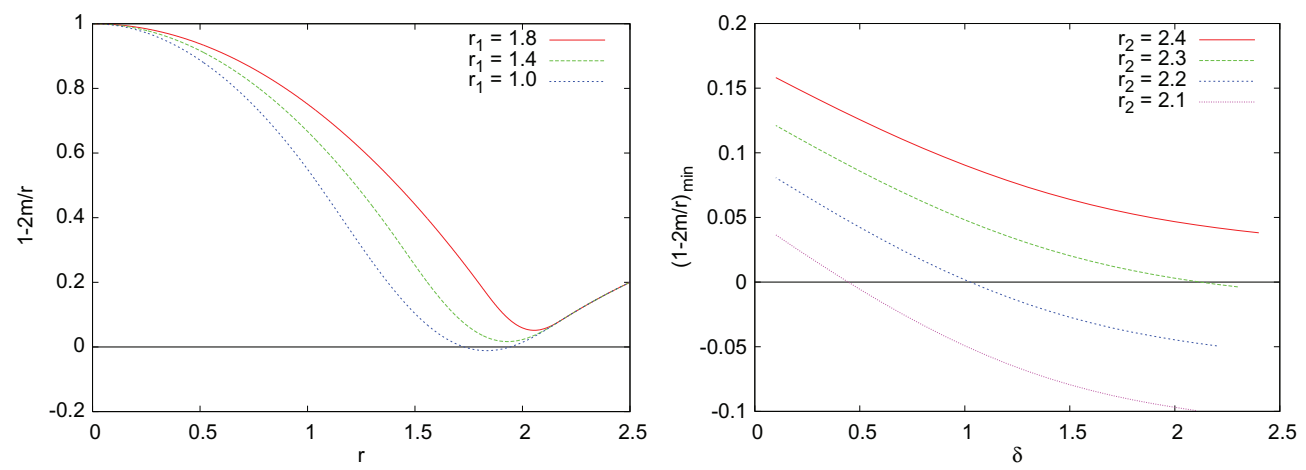

Figure 3. Left panel: behaviour of $1-2 m(r) / r$ for $M=1$ and different values of $r_{1}$ and $r_{2}=2.2$ Right panel: dependence on $\delta$ of the minimum value of $1-2 m(r) / r$ for gravastars with $M=1$ and different values of $r_{2}$. Note that for sufficiently large models, the minimum is always positive.

\subsection{Conditions on the metric functions}

While equations (22)-(31) allow us to construct gravastar models, additional constraints need to be imposed to guarantee that the metric functions $g_{t t}$ and $g_{r r}$ (and their first derivatives) have the expected properties once our choice for the EOS $p_{r}(\rho)$ and $\rho(r)$ is made. In particular, it is not difficult to conclude that $m(r)$ and $m^{\prime}(r)$ are continuous for the choice of $\rho(r)$ made in equation (23), so that using equation (17) $g_{r r}$ and its first derivative are continuous throughout the spacetime.

Similar considerations apply for the metric function $g_{t t}$, for which we note that in obtaining the solution for $v$ by integrating equation (18) we must allow for a constant of integration $v_{0}$ so that

$$
v=\int_{0}^{r} \frac{2 m(r)+8 \pi r^{3} p_{r}}{r(r-2 m(r))} \mathrm{d} r+v_{0} .
$$

The integration constant can then be determined through the condition that, at the surface of the gravastar, $g_{t t}\left(r_{2}\right)=-\left(1-2 M / r_{2}\right)$, thus giving

$$
g_{t t}=-\mathrm{e}^{v(r)}=-\left(1-\frac{2 M}{r_{2}}\right) \mathrm{e}^{\Gamma(r)-\Gamma\left(r_{2}\right)}
$$

where

$$
\Gamma(r) \equiv \int_{0}^{r} \frac{2 m(r)+8 \pi r^{3} p_{r}}{r(r-2 m(r))} \mathrm{d} r .
$$

In this way $g_{t t}$ and $g_{t t}^{\prime}$ are continuous across $r_{2}$ and throughout the spacetime.

The requirement for the metric functions of being continuous with continuous first derivatives is, in general, not sufficient to guarantee the existence of acceptable equilibrium models. The three free parameters $M, r_{1}$ and $r_{2}$, in fact, cannot be chosen arbitrarily but in such a way that $g_{r r}$ is always positive. In the left panel of figure 3, we show a typical example of the behaviour of the function $1-2 m(r) / r$, where different lines refer to different values of the inner radius $r_{1}$ while the outer one $r_{2}$ is held fixed. Clearly, an incorrect choice of $r_{1}$ and $r_{2}$ leads to negative values for $g_{r r}$ and thus to the unphysical appearance of horizons. The right panel of figure 3 shows how the minimum value of the function $1-2 m(r) / r$ depends on the gravastar's thickness $\delta$ and that gravastars with large thicknesses can be built if the outer radius $r_{2}$ is chosen to be sufficiently large, that is, if the compactness $\mu$ is sufficiently small. 


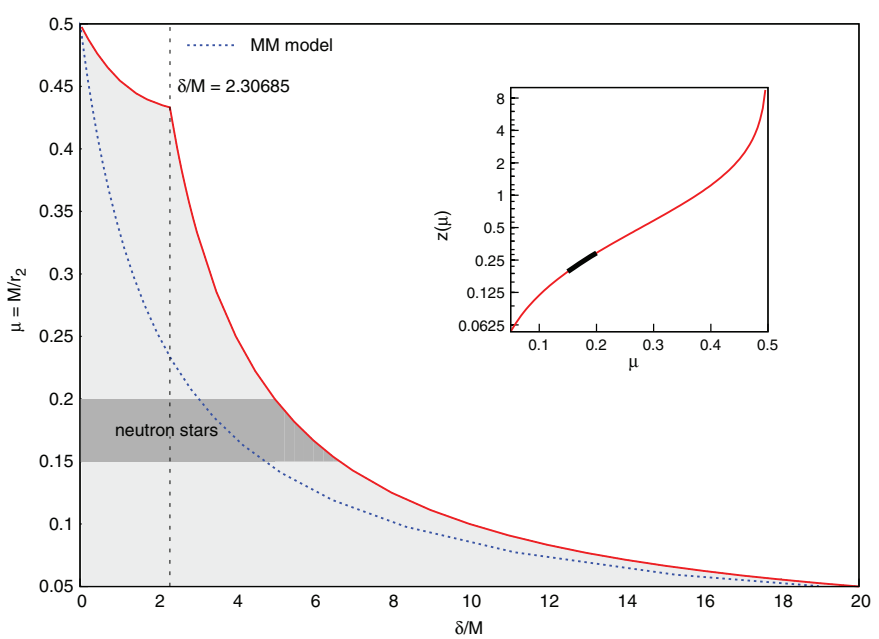

Figure 4. Limit on the compactness $\mu$ of the gravastar with the thickness of the shell $\delta$. This figure compares the results obtained for the MM model in the lower curve (see figure 1) and the results obtained for our model in the upper curve. The inset shows the surface redshift of the gravastar, $z$, as a function of the compactness $\mu$, with the thick black segment referring to the redshift range for standard neutron stars.

As a more systematic characterization of this problem, we have constructed a large number of gravastar models in which we have varied both the compactness $\mu$ and the thickness $\delta$. In this way we can extend the diagram presented in figure 1 for the existence of MM models to our thick-shell gravastar model and define the region in the $(\delta, \mu)$ parameter space where equilibrium solutions can be found. In particular, by defining the parameter $\epsilon$ as the dimensionless distance of the gravastar's surface from a Schwarzschild horizon, i.e., $\epsilon \equiv r_{2} / M-2$, we find that for $\epsilon>\epsilon_{c} \approx 0.3085$, the function $1-2 m(r) / r$ is always positive, so that the thickness $\delta$ can be as large as $r_{2}$ (i.e., $r_{1}$ can also be taken to be zero). On the other hand, for $\epsilon \leqslant \epsilon_{c}$ there exists a critical thickness $\delta_{c}=\delta_{c}\left(r_{2}, M\right)$ above which equilibrium models cannot be found because of the appearance of horizons. For $\epsilon=\epsilon_{c}$, we have found that $\delta_{c}=2.30685 M$.

Figure 4 summarizes these results by showing the region of the parameter space $(\delta, \mu)$ where equilibrium models can be calculated. Equilibrium solutions for our model can be found in the region below the solid line, and $\delta / M=2.30685$ is the boundary between the two distinct behaviours discussed above. Indicated with a dashed line is the corresponding threshold for the MM model (cf figure 1). Overall, the data in the figure reveal that our thick-shell model obeys similar but less restrictive bounds than the MM model. Also shown in the inset of figure 4 is the surface redshift of our gravastar model $z \equiv(1-2 \mu)^{-1 / 2}-1$ as a function of $\mu$. Clearly, no bound exists for this quantity and it is possible to build gravastars with arbitrarily large surface redshift, i.e., $z \rightarrow \infty$.

\section{Perturbation equations and numerical methods}

In order to assess the stability of gravastars against axial perturbations we have followed the standard procedure to study non-radial oscillations of stars [12, 13, 16, 17]. This approach differs from that adopted in [11], where the analysis of the axial perturbations of a gravastar was 
made following the standard procedure for Schwarzschild black holes (see [18] for a review) in order to study only the asymptotic properties of the potential and determine whether it has compact support and is everywhere positive.

We note that the pressure anisotropy in the shell does not play an important role in the study of axial tensor perturbations since these perturbations hardly excite fluid motions. As a result, the standard analysis for stars with isotropic pressures can be used here. This is no longer true for polar perturbations (for which the pressure anisotropy plays an important role) and a study on radial perturbations of stars with anisotropic pressures was recently presented in $[19,20]$.

As for Schwarschild black holes or spherical relativistic stars, the propagation of axial perturbations in the gravastar spacetime is governed by the wave equation in a scattering potential

$$
\frac{\partial^{2} \psi}{\partial r_{*}^{2}}-\frac{\partial^{2} \psi}{\partial t^{2}}=V_{\ell}(r) \psi
$$

where the 'tortoise' coordinate is defined as

$$
r_{*} \equiv \int_{0}^{r} \mathrm{e}^{(\lambda-v) / 2} \mathrm{~d} r
$$

and the scattering potential is given by the expression

$$
V_{\ell}(r) \equiv \frac{\mathrm{e}^{v}}{r^{3}}\left[\ell(\ell+1) r+4 \pi r^{3}\left(\rho-p_{r}\right)-6 m\right]
$$

The potential vanishes for $r \rightarrow \infty$ and diverges as $1 / r^{2}$ for $r \rightarrow 0$, as a result of the centrifugal term proportional to $\ell(\ell+1) / r^{2}$. This is an important difference that the potential $V_{\ell}(r)$ has with respect to its counterpart for a Schwarzschild black hole, where $V_{\ell}(r) \rightarrow 0$ at the horizon $r=2 M$. Note also that $\mathrm{e}^{v} \rightarrow 1$ for $r \rightarrow \infty$ (the spacetime is the Schwarzschild one for $\left.r>r_{2}\right)$ and, from equations (33) and (34), $\mathrm{e}^{v} \rightarrow\left(1-2 M / r_{2}\right) \mathrm{e}^{-\Gamma\left(r_{2}\right)}$ for $r \rightarrow 0$.

Unlike for black-hole spacetimes, both the tortoise coordinate and the scattering potential are here not expressed through simple algebraic relations but need to be computed as solutions of ordinary differential equations. We have done so using a fourth-order Runge-Kutta method to obtain $\mathrm{e}^{v}$ and $r_{*}$ from equations (33) and (36), respectively.

Introducing now the 'light-cone' (null) variables $u \equiv t-r_{*}$ and $v \equiv t+r_{*}$, the wave equation (35) can be written in the more compact form as

$$
-4 \frac{\partial^{2} \psi}{\partial u \partial v}(u, v)=V_{\ell}(r) \psi(u, v),
$$

and thus be integrated numerically. In particular, we have used a variation of the method used in [21] and in many other works, and derived a strict second-order accurate discretization given by

$$
\psi_{N}=\left(\psi_{E}+\psi_{W}\right) \frac{16-\Delta^{2} V_{S}}{16+\Delta^{2} V_{S}}-\psi_{S}+\mathcal{O}\left(\Delta^{4}\right),
$$

where the indices $N, S, E$ and $W$ refer to the grid points defined as $N \equiv(u+\Delta, v+\Delta), S \equiv$ $(u, v), E \equiv(u, v+\Delta)$ and $W \equiv(u+\Delta, v)$. Equation (38) was then numerically integrated in the $(u, v)$ plane with the algorithm (39) using a triangular grid limited by the lines $r_{*}=r_{*}^{\min }$ and $u=0$, where $r_{*}^{\min }$ is a small value which we have set as $r_{*}^{\min }=\Delta / 2$. A schematic representation of the null grid is presented in figure 5, where the black points represent the grid points where the solution is known, while the red ones are those where the solution is to be calculated. 


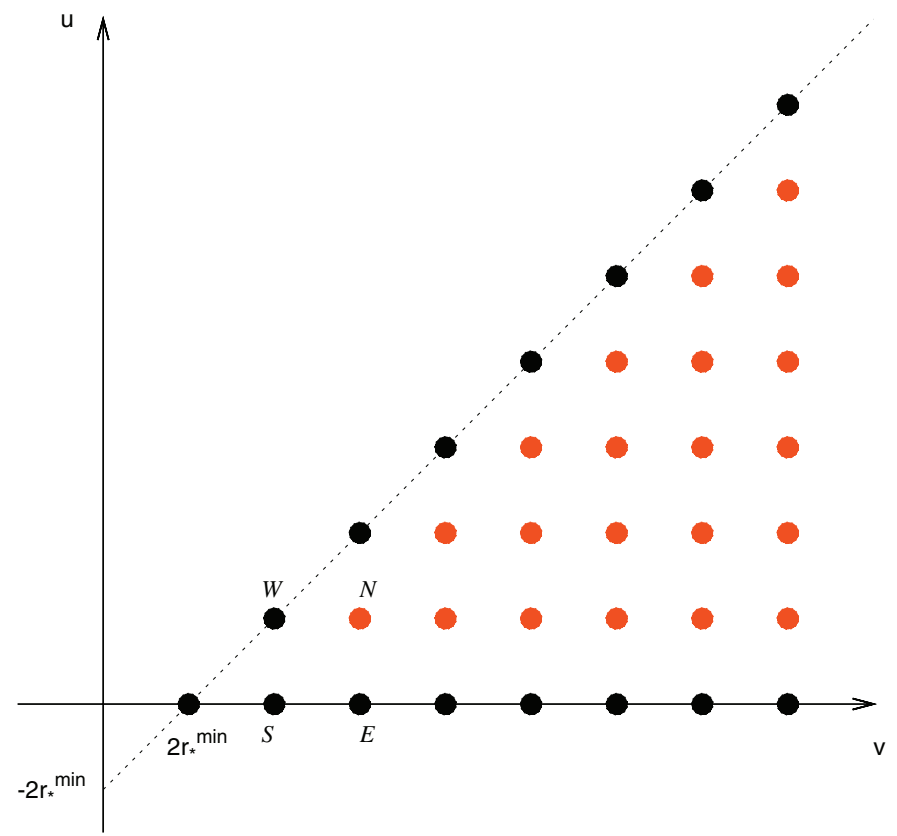

Figure 5. Diagram of the numerical grid and the domain of interest. The black points represent the grid points where the value of the field is known. The red points (online version only) represent the grid points to be calculated.

In a linear regime the eigenfrequencies of the gravastar are not sensitive to the choice of the initial data and thus we have modelled the initial perturbation with the simplest possible choice, i.e. a Gaussian pulse centred around $v_{c}$ and with width $\sigma$ :

$$
\psi\left(u=u_{0}, v\right)=\exp \left[-\frac{\left(v-v_{c}\right)^{2}}{2 \sigma^{2}}\right],
$$

where, to satisfy the regularity condition at the origin, we set

$$
\psi\left(r_{*}^{\min }, t\right)=\psi\left(u=v-v_{0}, v\right)=0, \quad \forall t .
$$

During the integration of equation (38) we extract the values of the field $\psi$ along a line of constant $r_{*}$, i.e., at the points $\left(u=v-2 r_{*}, v\right)$. We let the field evolve for large values of $t$, until the transient is over and possible contributions of the higher overtones have died off. Only then, when the signal consists of the fundamental mode (the slowest decaying mode) and of its overtones, we can compute the real $\omega_{R}$ and imaginary part $\omega_{I}$ of its eigenfrequency with a least-squares fit of the function

$$
\psi_{f i t}(t)=A_{n} \exp \left(\omega_{I}^{n} t\right) \cos \left(\omega_{R}^{n} t+\phi\right),
$$

where $A$ and $\phi$ are constant coefficients and $n$ represents the order of the mode and this is not meant as an exponent. Once the properties of the fundamental mode are known, the eigenfrequencies of the overtones are obtained using the method proposed in [22], which consists of subtracting from the numerical solution the fitted function relative to the fundamental mode $n=0$. This reveals the $n=1$ mode and the procedure can be iterated for obtaining higher overtones, depending on their damping rate. Of course, very rapidly decaying modes cannot be obtained by this procedure, but this approach is a very efficient and 

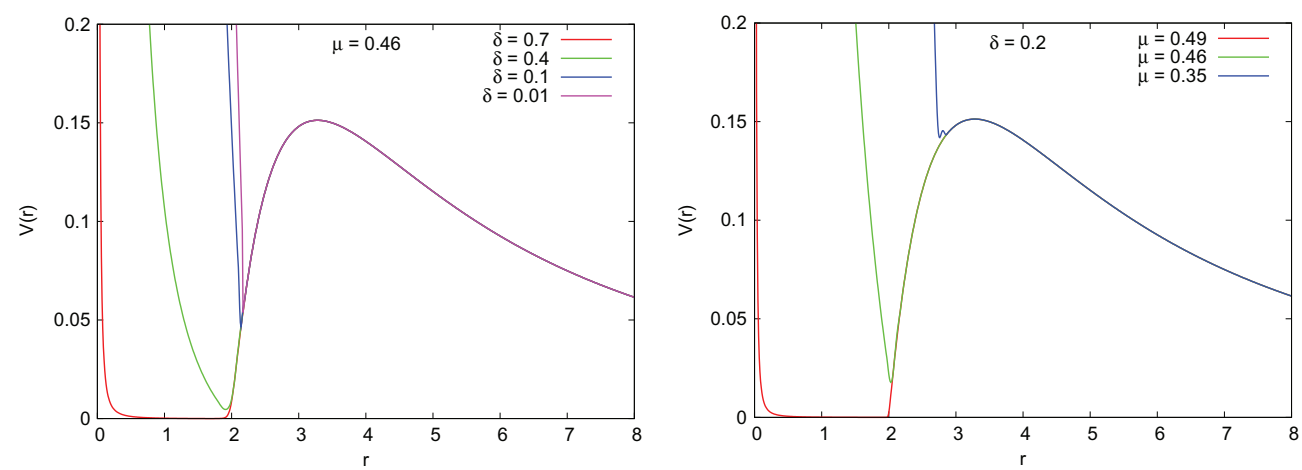

Figure 6. Left panel: the scattering potential $V_{\ell}(r)$ with $\ell=2$ of gravastar models with the same compactness $\mu=0.46$ but increasing thickness of the shell. Right panel: the same as in the left panel but gravastar models with the same shell thickness $\delta=0.2$ but increasing compactness.

easy way to obtain the first overtones in a signal, which are those expected to be detected from any astrophysical sources.

The numerical set-up described above has been first tested by performing a perturbation analysis of Schwarzschild stars, i.e., uniform-density spherical stars, obtaining results that are in very good agreement with the values reported in the literature [13, 23].

\section{Results}

Before discussing the results of the perturbative analysis, we note that the axial potential given in (37) shows features which are similar to those that are well known in compact uniformdensity stars [13]. Quite generically, in fact, the potential exhibits at least a minimum and a maximum, thus indicating the possibility of having modes that are trapped in the potential well.

In figure 6, we present some typical examples of the scattering potential $V_{\ell}(r)$ when $\ell=2$ and which, in general, depends on both the compactness $\mu$ (as for relativistic stars) and the thickness of the shell $\delta$. The left panel of figure 6 , in particular, shows the potential of gravastar models having the same compactness $\mu=0.46$ but increasing thickness of the shell, while the right panel reports how the potential changes for gravastar models with the same shell thickness $\delta=0.2$ but increasing compactness. Clearly, in the exterior region $V_{\ell}(r)$ is just the Schwarzschild potential for axial perturbations and the depth of the potential well in the interior region increases with both $\mu$ and $\delta$. Furthermore, given the chosen EOS (29), it is also possible to select the parameters $r_{1}, r_{2}$ and $M$ such that $V_{\ell}(r)$ has two local minima very close to each other. This is shown by the curve with $\mu=0.35$ in the right panel of figure 6 .

Following the procedure outlined in the previous section, we have integrated the perturbation equation (38) for a large variety of gravastar models differing in mass, compactness and thickness. Figure 7 reports some typical results and shows the time evolution of the perturbations for a representative gravastar with $M=1$ and radii $r_{1}=1.96, r_{2}=2.26$. The left panel, in particular, reports the total solution $\psi(t)$ as extracted at $r_{*}=10$ during the initial stages of the scattering off the potential and allows one to discern the 'beating' caused by the superposition of overtones and other rapidly decaying components of the signal. Interestingly, this beating of the fundamental mode with overtones becomes more pronounced with increasing $\mu$ and $\delta$, underlining that the contribution of the overtones increases (i.e., the 

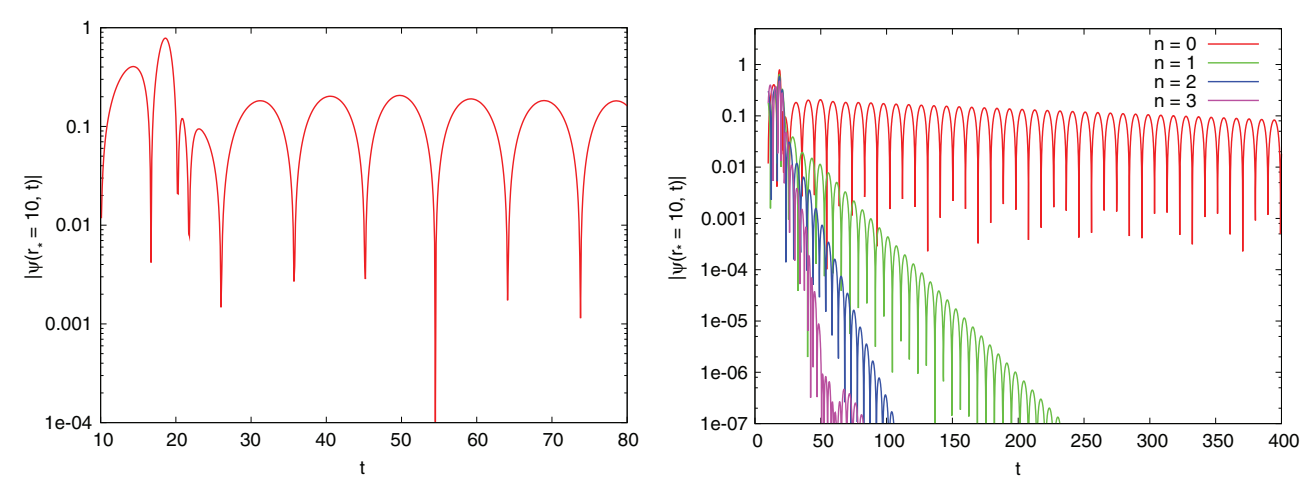

Figure 7. Left panel: the evolution of the axial perturbations $\psi(t)$ as extracted at $r_{*}=10$ and during the initial stages of the scattering for a gravastar with $M=1, r_{1}=1.96$ and $r_{2}=2.26$. Right panel: the same as in the left panel but when the contribution of the fundamental mode $n=0$ is distinguished from that of the overtones $n=1,2$ and 3 .

Table 1. Some typical values obtained for the first overtones of the gravastars QNM eigenfrequencies for axial perturbations with $\ell=2$. The gravastars have $M=1, r_{2}=2.26$ and $\delta$ given in the table. We also show for comparison the equivalent frequencies for a Schwarzschild black hole of the same mass and for a standard TOV star with uniform density with $M=1$ and $R=2.26 M$ (note that for these stars one must have $R / M>2.25$ [13]).

\begin{tabular}{|c|c|c|c|c|c|c|}
\hline \multirow[b]{2}{*}{ Model } & \multicolumn{2}{|c|}{$n=0$} & \multicolumn{2}{|c|}{$n=1$} & \multicolumn{2}{|c|}{$n=2$} \\
\hline & $\omega_{R}$ & $-\omega_{I}$ & $\omega_{R}$ & $-\omega_{I}$ & $\omega_{R}$ & $-\omega_{I}$ \\
\hline$\delta=0.30$ & 0.3281 & $2.481 \times 10^{-3}$ & 0.4865 & $6.264 \times 10^{-2}$ & 0.6534 & $1.590 \times 10^{-1}$ \\
\hline$\delta=0.35$ & 0.2943 & $7.081 \times 10^{-4}$ & 0.4459 & $3.202 \times 10^{-2}$ & 0.5922 & $1.093 \times 10^{-1}$ \\
\hline$\delta=0.40$ & 0.2575 & $1.543 \times 10^{-4}$ & 0.4011 & $1.227 \times 10^{-2}$ & 0.5384 & $5.814 \times 10^{-2}$ \\
\hline Schwarzchild black hole & 0.3737 & $8.896 \times 10^{-2}$ & 0.3467 & $2.739 \times 10^{-1}$ & 0.3011 & $4.783 \times 10^{-1}$ \\
\hline Schwarzchild star & 0.1090 & $1.239 \times 10^{-9}$ & 0.1484 & $3.950 \times 10^{-8}$ & 0.1876 & $5.470 \times 10^{-7}$ \\
\hline
\end{tabular}

modes decay more slowly) with both $\mu$ and $\delta$ (i.e., as the depth of the potential well increases). A similar relation between the decay rate of the modes and the potential well is known for the 'trapped modes' of compact uniform-density stars [23].

The right panel of figure 7 , on the other hand, shows the evolution of the perturbations over a longer timescale and when the overtones have been 'removed' from the general signal using the approach discussed in the previous section. Note that, for increasing $n$, the period of the oscillations becomes smaller and the decaying timescale shorter. Using a least-squares fit of the numerical data to the ansatz (42) we have computed the real and imaginary parts of the QNM eigenfrequencies and the numerical results for some typical cases are presented in table 1, where they are also compared to the corresponding values for a Schwarzschild black hole of the same mass and a Schwarzschild star of the same compactness. The tabulated values are all for an $\ell=2$ perturbation and refer to the fundamental mode and to the first two overtones since the third one does not usually have a sufficiently clear slope to allow for an accurate measurement. If necessary, an increase in spatial resolution would improve the decay of higher overtones and allow for their computation.

Figure 8 is the most important in this paper and it reports the variation of the $\ell=2, n=0$ QNM eigenfrequencies as a function of the parameters of the gravastar: $\mu$ and $\delta$. The two 

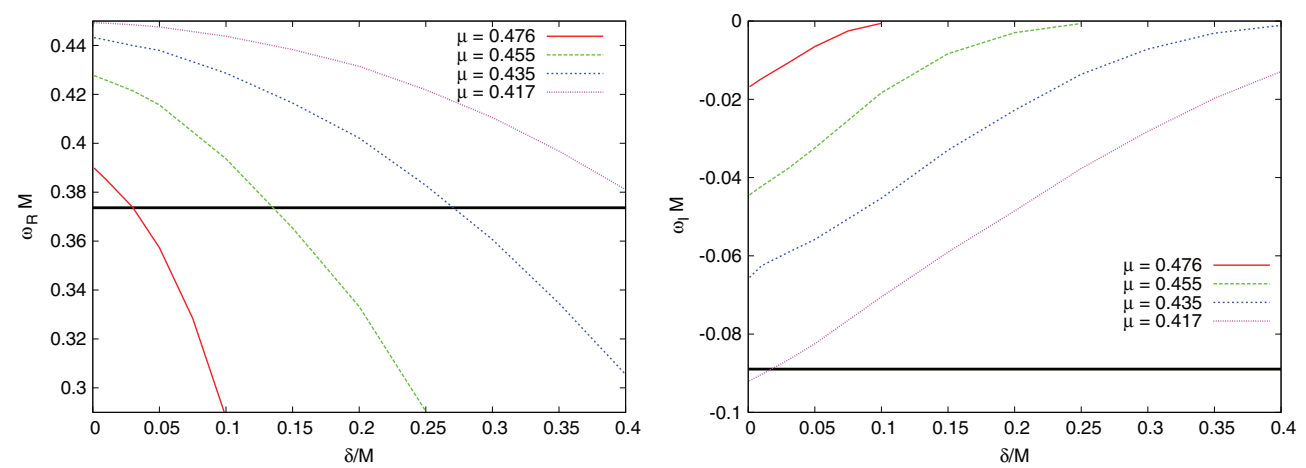

Figure 8. Left panel: behaviour of the real part $\omega_{R}$ of the $\ell=2, n=0$ QNM eigenfrequencies as functions of $\delta$ for different values of $\mu$. Right panel: the same as the left panel but for the imaginary part $\omega_{I}$ of the eigenfrequencies. In both panels, the thick horizontal lines represent the corresponding frequencies for a Schwarzschild black hole.

panels separately report the behaviour of the real and imaginary parts and indicate that both the period of the oscillations $\tau_{R} \equiv 2 \pi / \omega_{R}$ and the damping time $\tau_{I} \equiv 2 \pi /\left|\omega_{I}\right|$ increase with increasing $\delta$ and $\mu$. The two thick horizontal lines instead represent the corresponding frequencies for a Schwarzschild black hole of the same mass, i.e., $M \omega_{R}=0.37367$ and $M \omega_{I}=-0.08896$ [16]. Clearly, while it is always possible to select the thickness and compactness of the gravastar such that it will have the same oscillation frequency of a black hole with the same mass (cf left panel), the corresponding decaying time will be different and about one order of magnitude larger (cf right panel). Stated differently, a gravastar and a black hole with the same mass cannot have the same complex QNM eigenfrequency when subject to axial perturbations. This result, which we have presented here for the fundamental mode, can be shown to be true also for the first two overtones.

\section{Conclusions}

Although the gravastar model of Mazur and Mottola [1] represents an ingenious solution of the Einstein equations in spherical symmetry, it has also challenged one of the most cherished foundations of modern astrophysics, i.e., the existence of astrophysical black holes. Gravastars, in fact, can be constructed to be arbitrarily compact, with an external surface which is only infinitesimally larger than the horizon of a black hole with the same mass. As a result, the electromagnetic emission from the surface of a gravastar will suffer from essentially the same gravitational redshift as that of a black hole, thus making it difficult, if possible at all, to distinguish the two when only electromagnetic radiation is available.

Without entering the relevant debate about the physical processes that would lead to the formation of a gravastar or the astronomical evidence in support of their existence [24], we have here considered two more fundamental questions: is a gravastar stable against generic perturbations? If so, can an external observer distinguish it from a black hole? The short answers to these questions are that a gravastar is stable to axial perturbations and indeed it is possible to distinguish it from a black hole if gravitational radiation is produced.

To reach the first of these conclusions we have constructed a general class of gravastar models that extends the one proposed by Mazur and Mottola by replacing the infinitesimal shell of matter with one having finite size $\delta$ and variable compactness $\mu$. These equilibrium 
solutions of the Einstein equations have then been analysed when subject to axial perturbations and the eigenfrequencies of the corresponding QNMs have been computed explicitly. For all of the cases considered, the imaginary part of the eigenfrequencies has always been found to be negative, thus indicating the stability of these objects with respect to this type of perturbations.

To reach the second conclusion, instead, we have shown that the QNM spectra of a gravastar and that of a black hole of the same mass differ considerably. In particular, while it is always possible to select $\delta$ and $\mu$ such that the gravastar has the same oscillation frequency as that of a black hole with the same mass, the corresponding decaying time will be different. As a result, the gravitational radiation produced by an oscillating gravastar can be used to distinguish it, beyond dispute, from a black hole of the same mass.

We plan to extend our stability analysis also to polar perturbations and determine whether or not these intriguing objects possess modes of oscillation that do not have a counterpart in compact relativistic stars and may therefore hint to new solutions of the Einstein equations.

\section{Acknowledgments}

LR dedicates this work to Richard A Matzner on the occasion of his 65th birthday. Support comes from the Fundação de Amparo à Pesquisa no Estado de São Paulo (FAPESP), the Deutsche Akademische Austauschdienst (DAAD) and the Max-Planck-Gesellschaft (MPG).

\section{References}

[1] Mazur P O and Mottola E 2001 Gravitational condensate stars: an alternative to black holes Preprint gr-qc/0109035

[2] Chapline G 2004 Dark energy stars Proc. 22nd Texas Conf. on Relativistic Astrophysics (CA) Mazur P O and Mottola E 2004 Gravitational vacuum condensate stars Proc. Natl Acad. Sci. 111 9545-50

[3] Israel W 1966 Singular hypersurfaces and thin shells in general relativity Nuovo Cimento B 44 1-14 Israel W 1967 Nuovo Cimento B 48463 (erratum)

Misner C W, Thorne K S and Wheeler J A 1997 Gravitation (New York: Freeman) pp 505-16, 551-6

[4] Abramowicz M A, Kluzniak W and Lasota J P 2002 No observational proof of the black-hole event-horizon Astron. Astrophys. 396 L31-4

[5] Visser M and Wiltshire D L 2004 Stable gravastars - an alternative to black holes? Class. Quantum Grav. 21 $1135-51$

[6] Carter B M N 2005 Stable gravastars with generalised exteriors Class. Quantum Grav. 22 4551-62

[7] Bilić N, Tupper G B and Viollier R D 2006 Born-Infeld phantom gravastars J. Cosmol. Astropart. Phys. JCAP2(2006)13

[8] Lobo F S N 2006 Stable dark energy stars Class. Quantum Grav. 23 1525-41

[9] Lobo F S N and Arellano A V B 2007 Gravastars supported by nonlinear electrodynamics Class. Quantum Grav. 24 1069-88

[10] Cattoen C, Faber T and Visser M 2005 Gravastars must have anisotropic pressures Class. Quantum Grav. 22 4189-202

[11] DeBenedictis A, Horvat D, Ilijic S, Kloster S and Viswanathan K S 2006 Gravastar solutions with continuous pressures and equation of state Class. Quantum Grav. 23 2303-16

[12] Chandrasekhar S and Ferrari V 1991 On the non-radial oscillations of a star Proc. R. Soc. A 432 247-79

[13] Chandrasekhar S and Ferrari V 1991 On the non-radial oscillations of a star: III. A reconsideration of the axial modes Proc. $R$. Soc. A 434 449-57

[14] Dymnikova I 1992 Vacuum nonsingular black hole Gen. Rel. Grav. 24 235-42

Dymnikova I and Galaktionov E 2005 Stability of a vacuum nonsingular black hole Class. Quantum Grav. 22 2331-57

[15] Mbonye M R and Kazanas D 2005 A non-singular black hole model as a possible end-product of gravitational collapse Phys. Rev. D 72 024616-23

[16] Kokkotas K D and Schmidt B G 1999 Quasi-normal modes of stars and black holes Living Rev. Rel. 22

[17] Nollert H 1999 Quasinormal modes: the characteristic 'sound' of black holes and neutron stars Class. Quantum Grav. 16 R159-216 
[18] Nagar A and Rezzolla L 2005 Gauge-invariant non-spherical perturbations of Schwarzschild black-hole spacetimes Class. Quantum Grav. 22 R167-92

Nagar A and Rezzolla L 2006 Gauge-invariant non-spherical perturbations of Schwarzschild black-hole spacetimes Class. Quantum Grav. 234297 (erratum)

[19] Dev K and Gleiser M 2002 Anisotropic stars: exact solutions Gen. Rel. Grav. 34 1793-818

[20] Dev K and Gleiser M 2003 Anisotropic stars: 2. Stability Gen. Rel. Grav. 35 1435-57

[21] Abdalla E, Chirenti C B M H and Saa A 2006 Quasinormal modes for the Vaidya metric Phys. Rev. D 74 084029-34

[22] Abdalla E and Giugno D 2006 An extensive search for overtones in Schwarzschild black holes Preprint gr-qc/0611023

[23] Kokkotas K D 1994 Axial modes for relativistic stars Mon. Not. R. Astron. Soc. 268 1015-8

Kokkotas K D 1995 Axial modes for relativistic stars Mon. Not. R. Astron. Soc. 277277 (erratum)

Abdalla E and Giugno D 2006 Shedding some new lights upon the stellar quasi-normal modes Preprint gr-qc/0612183

[24] Broderick A E and Narayan R 2007 Where are all the gravastars? Limits upon the gravastar model from accreting black holes Class. Quantum Grav. 24 659-66 